\title{
Investigación
}

\section{El poder del}

algoritmo y

la vida social

DOI: 10.29236/sistemas.n161a4

Andrés R. Almanza J.

Estamos en un mundo cada vez más movilizado desde el contexto digital y, por tanto, es tarea de todos poder entender que los algoritmos forman parte de la realidad que nos rodea.

\section{Resumen}

El presente documento recoge algunas ideas en relación con el progreso de los algoritmos como fuente significativa de impacto en la sociedad, desde el uso de estos en la vida cotidiana a través de las redes sociales, hasta la forma en cómo los gobiernos, estados y naciones los usan para movilizar a las organizaciones. Este artículo no está diseñado para explorar todos los aspectos del poder de los algoritmos, sino para dar algunas luces sobre aquello que está sucediendo en el desarrollo de una vida digital tan acelerada como la que tenemos en la actualidad.

\section{Palabras clave}

Redes sociales, desinformación, manipulación, algoritmos, 


\section{Introducción}

Las dinámicas complejas que actualmente vive la humanidad a raíz de la pandemia producida por el COVID-19, ha transformado todos los aspectos de la vida, en lo cotidiano y lo que no lo es; ha acrecentado y acelerado las dinámicas y complejidad que el mundo esperaba, pero no con saltos cuánticos, a los que la sociedad en todas sus dimensiones ha visto impactos $y$ efectos inesperados, para los cuales no existía preparación.

La incertidumbre es una tendencia que hoy por hoy ha tomado fuerza y se convierte en una herramienta que modelará no solo el 2021, sino los años venideros de acuerdo con el reciente reporte del CIDOB (Soler, 2020), al resaltar que los movimientos que verá el mundo en todas sus dimensiones.

Los algoritmos siempre han sido una parte esencial de nuestra vida profesional, personal y social; en todas las verticales e industrias de la edad moderna, han facilitado el desarrollo de la ciencia naturales, sociales y las humanidades y, de paso, han ayudado a enriquecer la vida con conocimiento. Así mismo, han permitido que la tecnología lleve más allá de los límites el desarrollo. (Abiteboul. S., Dowek. G., 2020)

En consecuencia, este documento, pretende revisar algunos reportes en relación con las implicaciones que están teniendo los algoritmos en la vida de las personas, naciones y negocios, y cómo sus efectos vienen creando temas que se instalan en las agendas globales, como la desinformación, las adicciones a los medios sociales y los procesos electorales de las sociedades.

\section{El poder de un algoritmo}

Hablar del poder de las máquinas es hablar sobre cómo la tecnología ha evolucionado en el tiempo; es hablar del desarrollo en sí mismo y de lo que ha traído para las sociedades y como fin último a las personas.

La actualidad está modelada por servicios digitales basados en algoritmos, que no son otra cosa que capacidades computacionales, hoy más potentes (inteligentes) que procesan datos, con un impacto en todos los aspectos de la vida de las personas (Ausiello., G., Rosella. P., 2013).

Los algoritmos han venido cambiando las dinámicas sociales y culturales en las últimas décadas (Beer.D., 2016) y en los tiempos acelerados como los actuales, han aumentado su nivel de presencia y exposición.

La vida como la conocemos está influenciada por la vida digital, los servicios digitales y el consumo de la información, y la tecnología es una forma de materializar los intereses de un conglomerado; por tanto, no podría existir la tecnología sin una necesidad y ésta no podría ser 
materializada sin datos para procesar (Sadowski. J., 2020).

Para este documento se han declarado frentes en que los algoritmos han tenido una notoria presencia. La desinformación, la vida social de las personas y la manipulación de los medios sociales.

\section{Democracias expuestas a la} manipulación digital

Manipulación social hostil, es considerado el término que desde el 2016 engloba a la desinformación, la propaganda, la manipulación de la información y las noticias falsas (Mazarr. M., et al., 2019), que han mostrado el poder de los algoritmos detrás de las intenciones, de estados, naciones, organizaciones, grupos políticos y otros como formas de crear nuevos conflictos acordes con sus propios intereses.

Los medios sociales están jugando un papel importante en compartir este tipo de información, por su poder expandido alrededor del mundo; estas tecnologías han creado grandes beneficios para la humanidad y también grandes desafíos.

Las "cyber troops", definidas como los gobiernos o partidos políticos han desarrollado la manipulación de la opinión publica en el contexto digital (Bradshaw, S., et al., 2020)

En el informe titulado "La industrialización de la desinformación" (Bradshaw, S., et al., 2020), son tres las principales tendencias que existen:

1. Continúan y continuarán siendo usadas estas tendencias de maneraglobal.

Se ha logrado identificar que son cerca de 81 países según monitoreo oficial los que usan este tipo de tácticas, comparado con años anteriores que solo estaban registrados 70 (Figura 1).

2. Se intensifica el trabajo económico y el combate contra el fenómeno.

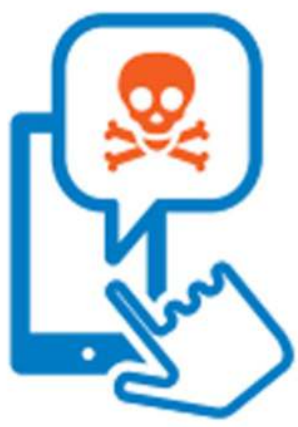

81 countries

use social media to

spread computational

propaganda and

disinformation

Figura 1 Cantidad de actores. Fuente: https://demtech.oii.ox.ac.uk/wpcontent/uploads/sites/127/2021/02/CyberTroop-Report20-Draft9.pdf 


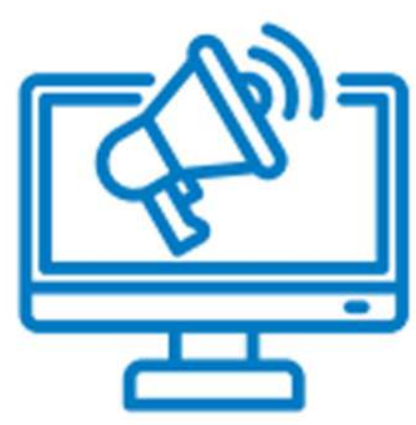

almost

us $\$ 10^{\mathrm{M}}$

spent on political

adverts by cyber

troops operating

world wide

Figura 2. Monto invertido. Fuente: https://demtech.oii.ox.ac.uk/wpcontent/uploads/sites/127/2021/02/CyberTroop-Report20-Draft9.pdf

Se tiene evidencia de que todas las compañías hacen esfuerzos por limitar, controlar, y reducir en sus plataformas este tipo de actividades. Según datos del informe, se registra que Facebook ha eliminado entre 2019 y 2020, más de 10,800 cuentas, 12,500 páginas, 603 grupos, 1556 cuentas de Instagram, y más de 290.000 cuentas de Twitter. De la misma manera se reportan cerca de \$US 10 millones de dólares gastados en esto, según cifras de Facebook (Figura 2).

\section{countries with evidence of \\ private firms managing manipulation campaigns}

3. Creciente y significativo negocio. Cada vez más existen empresas que se forman para la prestación de los servicios de manipulación de compañas políticas; existen firmas privadas -alrededor de 48 en todo el mundo-, las cuales usan desde bots, microsegmentación de público, creación de cuentas marionetas (que no se consideran falsas). El negocio puede estar alrededor de \$US60 Millones de dólares de inversión para lograr el objetivo del servicio (Figura 3).

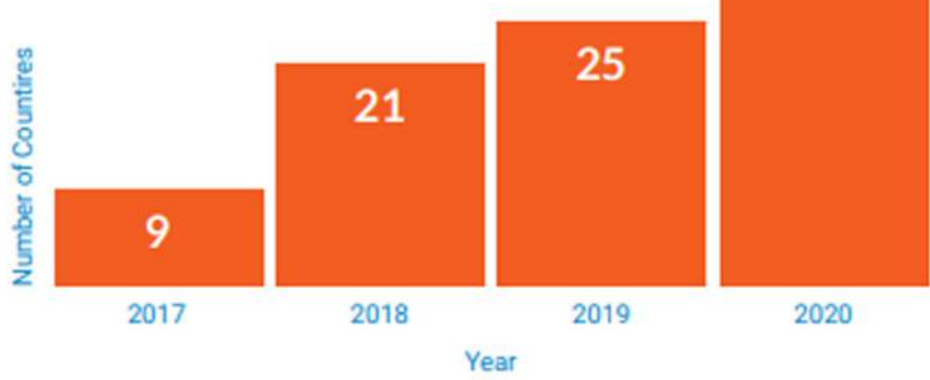




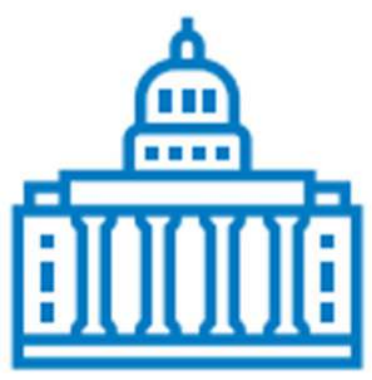

\section{US $\$ 60^{\mathrm{M}}$}

spent by state actors on contracts with private firms for computational propaganda services

Figura 3. Presencia empresas. Fuente: https://demtech.oii.ox.ac.uk/wpcontent/uploads/sites/127/2021/02/CyberTroop-Report20-Draft9.pdf

El uso por parte de las agencias de gobierno crece; el informe evidencia que, por lo menos, en 62 países se usa este tipo de situaciones para direccionar la intención del público; aspectos como la propaganda computacional es el instrumento mayormente usado. En esta categoría se encuentran desde ministros digitales, campañas militares o actividad de las fuerzas de policía.

Los partidos políticos no se quedan atrás, según el informe hay evidencia de que en, por lo menos 61 países de los 80 revisados, algunos partidos políticos utilizan este tipo de estrategias digitales, en la mayoría de los casos para afectar a contendores políticos.

Si bien el informe está centrado en lo que hacen los gobiernos y los partidos políticos en los países estudiados, no significa que otros actores no usen las redes sociales para realizar este tipo de situaciones (gráfica 4).

La gráfica 4, muestra que en países de la región LATAM, Colombia Bra- sil, Argentina y Ecuador tienen el mismo comportamiento, y que las agencias del gobierno, los partidos políticos y contratistas privados están haciendo uso de este tipo de herramientas.

En este mismo sentido las estrategias, técnicas y tácticas usadas son muy variadas, se adaptan de acuerdo con las necesidades del momento; sin embargo, se han identificado tres tipos de estrategias:

1. Cuentas. Es común observar el uso de cuentas de perfiles falsos como reales, y en ellas se pueden dar dos modelos: cuentas automáticas y cuentas cuyo contenido es curado, a través de las cuales se distribuye el mensaje para producir desinformación. El tercer mecanismo, son cuentas que previamente han sido hackeadas o robadas (Figura 5).

Para el caso de los países de la región LATAM, se encuentra todos los tipos, desde Bots, Humanas y Robadas o Hackeadas (Figura 6). 


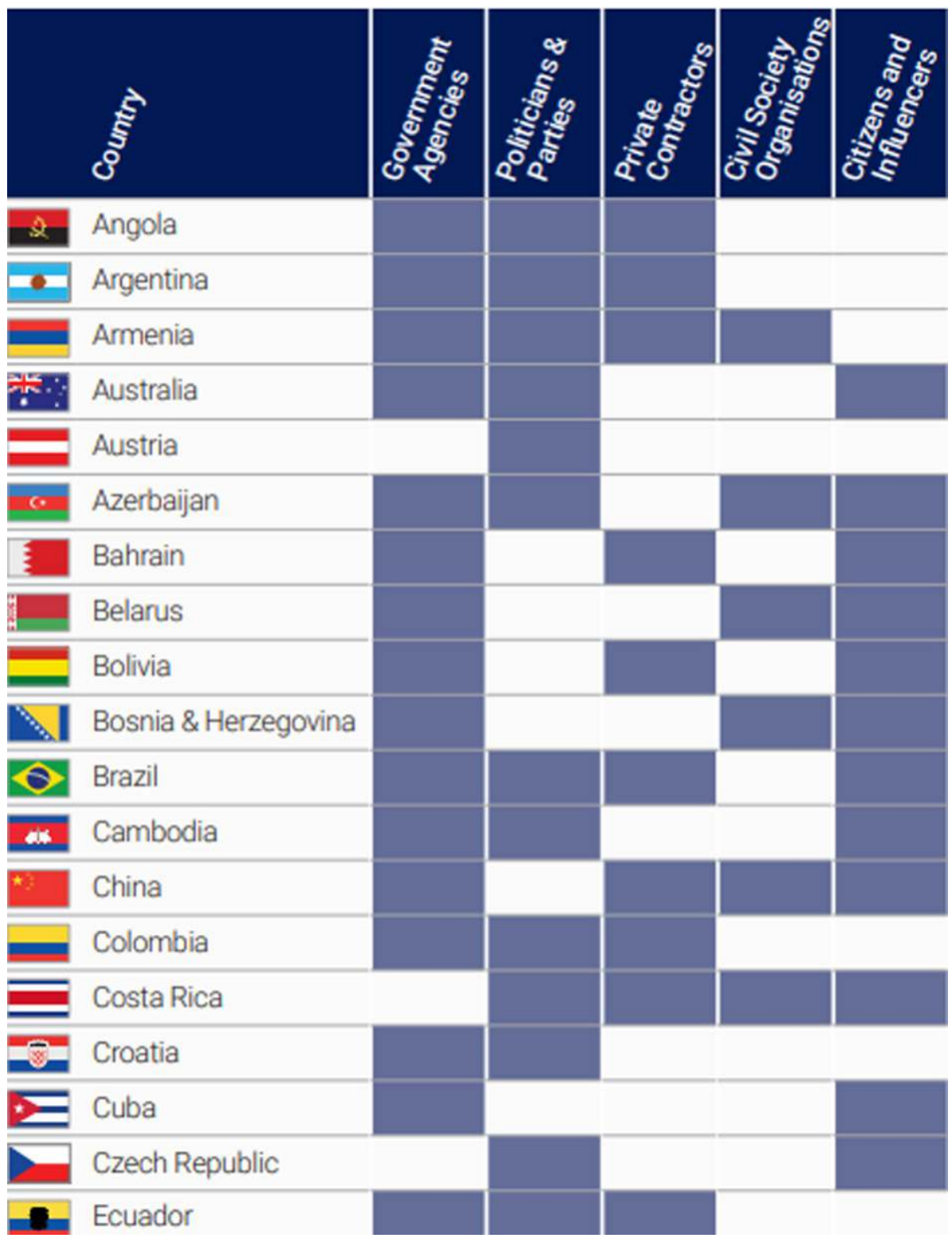

Gráfica 4 Países. Fuente: https://demtech.oii.ox.ac.uk/wpcontent/uploads/sites/127/2021/02/CyberTroop-Report20-Draft9.pdf

2. Mensaje y adherencia. Existen los opositores, supresión de la parmuchas formas de transmitir el ticipación a través del acoso, menmensaje y su atracción o repulsión sajes para dividir y polarizar a la poa través de los distintos medios, blación (Tabla 1). identificando cuatro tipos de mensajes, propaganda a favor del gobierno o partido político, ataques a

Según los datos evidenciados se encuentra que en todos los países 


\section{由 57 \\ countries used automated accounts}

\section{․ 79 \\ countries used human-curated accounts

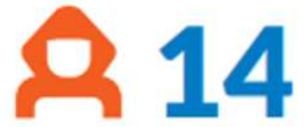 \\ countries used hacked, stolen or impersonation accounts}

Figura 5. Tipos de cuentas usadas. Fuente: https://demtech.oii.ox.ac.uk/wpcontent/uploads/sites/127/2021/02/CyberTroop-Report20-Draft9.pdf

los tipos de mensajes son usados, dejando en primer lugar los que atacan a la oposición, seguidos de los que apoyan al gobierno, luego los que buscan silenciar y, por último, los que suelen dividir (Figura $7)$.

Según el estudio, se puede evidenciar que los países de la región LATAM, usan todos los tipos de mensajes (Figura 8).
3. Técnicas y tácticas. Las estrategias de comunicación han sido clasificadas en cuatro tipos: creación de desinformación, estrategias basadas en datos, acoso, doxing (divulgación) y trolling (bombardeos) y denuncia masiva de contenidos (Tabla 2).

Los datos, confirman que de los países revisados todos usan por lo menos algún tipo de estas técnicas

\section{Tabla 1}

Tipos de mensajes

\begin{tabular}{|l|l|}
\hline Tipo de mensaje & Descripción \\
\hline Propaganda pro (gobierno, partido político) & $\begin{array}{l}\text { Se utiliza el poder computacional para dar } \\
\text { apoyo al gobierno o al partido político }\end{array}$ \\
\hline Ataque a los opositores & $\begin{array}{l}\text { Se usa regularmente con la difamación, o } \\
\text { cualquier tipo de mensaje que afecte a la } \\
\text { parte contraria. }\end{array}$ \\
\hline Supresión de la participación & $\begin{array}{l}\text { Se usa el acoso, los señalamientos para } \\
\text { hacer que las personas que no están en la } \\
\text { misma línea dejen de participar. }\end{array}$ \\
\hline Mensajes de división y polarización & $\begin{array}{l}\text { Mensajes que buscan que la población } \\
\text { general tome una de las dos ideas que se } \\
\text { plantean, que se dividan e incluso haya } \\
\text { conflictos por ello. }\end{array}$ \\
\hline
\end{tabular}

Nota: Elaboración propia 


\begin{tabular}{|c|c|c|c|c|}
\hline & Country & Bots & Human & $\begin{array}{l}\text { Hacked or } \\
\text { Stolen }\end{array}$ \\
\hline & Angola & |' & $\therefore$ & 8 \\
\hline 8 & Argentina & 茴| & 2 & 8 \\
\hline & Armenia & 䗆 & 2 & 8 \\
\hline$\%$ & Australia & 官1 & 2 & 8 \\
\hline & Austria & 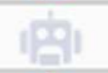 & 2 & 8 \\
\hline & Azerbaijan & 恕 & ㄷ. & 8 \\
\hline & Bahrain & 咸1 & 2 & 8 \\
\hline & Belarus & 189 & 돈 & 8 \\
\hline & Bolivia & 亩| & 2 & 8 \\
\hline 21 & Bosnia \& Herzegovina & 福 & ㄷ. & 8 \\
\hline$\theta$ & Brazil & 姆。 & 0 & 8 \\
\hline 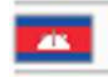 & Cambodia & 晏 & 2 & 8 \\
\hline$m$ & China & 皿 & $\therefore$ & 8 \\
\hline & Colombia & 为 & ㄷ. & 8 \\
\hline 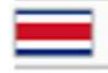 & Costa Rica & 189 & $\stackrel{2}{2}$ & 8 \\
\hline 줄 & Croatia & 酔 & 2 & 8 \\
\hline$=$ & Cuba & 㛾 & 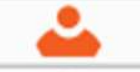 & 2 \\
\hline 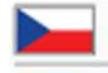 & Czech Republic & 蝠 & ․․ & 8 \\
\hline \pm & Ecuador & 䡒 & 2 & 2 \\
\hline
\end{tabular}

Figura 6. Países y tipos de cuentas. Fuente: https://demtech.oii.ox.ac.uk/wpcontent/uploads/sites/127/2021/02/CyberTroop-Report20-Draft9.pdf

(Ver figura 9); así mismo, la región LATAM muestra que en sus países este tipo de estrategias son ampliamente usadas (Ver figura 10).

4. Organización, capacidades y presupuestos. Estos grupos tienen unas estructuras, presupuestos y capacidades bien definidas. Sin embargo, varían dependiendo del país en donde se desarrollan. En cuanto a los presupuestos, dado que es un negocio lucrativo, se observa que hay rubros importantes designados para ello, y la capacidad está muy asociada a los criterios anteriores, dividida en tres grupos (Tabla 3). 


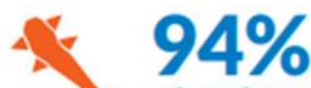

of study countries have misinformation campaigns that attack the opposition and mount smear campaigns

\section{$73 \%$}

of study countries have misinformation campaigns that suppress participation through trolling or harassment
$90 \%$

of study countries have misinformation campaigns that involve pro-government and pro-party propaganda

\section{$48 \%$}

of study countries have misinformation campaigns that drive division and polarize citizens

Figura 7. Porcentajes de tipos de mensajes. Fuente: https://demtech.oii.ox.ac.uk/wpcontent/uploads/sites/127/2021/02/CyberTroop-Report20-Draft9.pdf

La figura 11, muestra algunas muestras de países con alta, media y baja capacidad. Cabe resaltar que, de los estudiados, Venezuela es el único país considerado con alta capacidad para operar.

\section{Efectos de los medios digitales} en la infancia y adolescencia en Colombia

En los tiempos actuales de penetración de los medios digitales en las vidas de todas las personas, en especial las poblaciones de niños, niñas y adolescentes (NNA) cobra importancia revisar el efecto que el consumo de contenidos tiene en este grupo de población, más vulnerable.

En los últimos años se vienen haciendo cuestionamientos a las plataformas y medios digitales, redes sociales y otros, sobre el poco o nu-

Tabla 2

Técnicas, tácticas y estrategias

\begin{tabular}{|l|l|}
\hline Técnicas y tácticas & Descripción \\
\hline Creación de desinformación & $\begin{array}{l}\text { En esta categoría están todos los tipos de } \\
\text { "noticias falsas" noticias falsas. }\end{array}$ \\
\hline Estrategia basada en datos & $\begin{array}{l}\text { Usado para perfilar y segmentar a la } \\
\text { población con mensajes políticos } \\
\text { específicos. }\end{array}$ \\
\hline Bombardeo, Acoso, divulgación & $\begin{array}{l}\text { Redes creadas para estar en continua } \\
\text { operación y crear en los blancos definidos } \\
\text { efectos. }\end{array}$ \\
\hline Denuncias masivas & $\begin{array}{l}\text { A través de redes creadas se busca } \\
\text { reportar que algún tipo de contenido no es } \\
\text { adecuado, buscando que los medios } \\
\text { sociales lo pongan fuera de línea }\end{array}$ \\
\hline
\end{tabular}

Nota: Elaboración propia 


\begin{tabular}{|c|c|c|c|c|c|c|}
\hline & 今 & हैं & 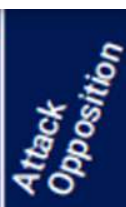 & 胥 & 今 & $e^{\delta}$ \\
\hline 2 & Angola & it & $x$ & $F$ & $4 x$ & $\otimes$ \\
\hline E- & Argentina & מל & 1 & $F$ & $4 x$ & $\otimes$ \\
\hline$E$ & Armenia & מ & 1 & $\vec{F}$ & $4 x$ & $\otimes$ \\
\hline Fis: & Australia & ط' & 1 & $\vec{F}$ & $4 x$ & $\otimes$ \\
\hline & Austria & it & $x$ & $F$ & $4 x$ & $\otimes$ \\
\hline & Azerbaijan & מל & 1 & $F$ & $4 x$ & $\otimes$ \\
\hline & Bahrain & מ & 1 & $F$ & $4 x$ & $\otimes$ \\
\hline & Belarus & H & $x$ & $\vec{F}$ & $4 x$ & $\theta$ \\
\hline & Bolivia & מל & 1 & $F$ & $4 x$ & $\otimes$ \\
\hline 21 & Bosnia \& Herzegovina & מ & $x$ & $F$ & $4 x$ & $\otimes$ \\
\hline 8 & Brazil & H & $x$ & $F$ & $4 x$ & $\otimes$ \\
\hline ats & Cambodia & It & 1 & $F$ & $4 x$ & $\otimes$ \\
\hline & China & מ & 1 & $F$ & $4 x$ & $\otimes$ \\
\hline & Colombia & 1) & 1 & $F$ & $4 x$ & $\otimes$ \\
\hline & Costa Rica & ith & 1 & $F$ & $4 x$ & $\otimes$ \\
\hline 조을 & Croatia & מ. & 1 & $\vec{F}$ & $4 x$ & $\otimes$ \\
\hline$\equiv$ & Cuba & מ & 1 & $F$ & $4 x$ & $\otimes$ \\
\hline$\square$ & Czech Republic & מו & 1 & $F$ & $4 x$ & $\otimes$ \\
\hline 2 & Ecuador & it & $x$ & $F$ & $4 x$ & $\otimes$ \\
\hline
\end{tabular}

Figura 8. Uso de tipos de mensajes por países. Fuente:

https://demtech.oii.ox.ac.uk/wp-content/uploads/sites/127/2021/02/CyberTroopReport20-Draft9.pdf

lo control que tienen sobre el nivel portancia que se le presta al tema de contenidos difundidos en las redes sociales; en la más reciente situación se encuentran las denuncias realizadas contra la red social Facebook, en particular con Instagram por los peligros y la poca imen cuestión.

En una reciente investigación del Washington Post, titulada "The Facebook Files" (WSJ), que contempla un compendio de documentos y 
Tabla 3

Clasificación de capacidad.

\begin{tabular}{|l|l|}
\hline Tipos de capacidad & Descripción \\
\hline Alta capacidad & $\begin{array}{l}\text { Disponen de grandes cantidades de } \\
\text { recursos, humanos, financieros, que } \\
\text { también involucra investigación y } \\
\text { desarrollo de nuevas tácticas }\end{array}$ \\
\hline Mediana capacidad & $\begin{array}{l}\text { Una capacidad estratégica para } \\
\text { coordinar esfuerzos, con menos } \\
\text { recursos. }\end{array}$ \\
\hline Baja capacidad & $\begin{array}{l}\text { Grupos pequeños, que son activados en } \\
\text { procesos temporales como los } \\
\text { electorales, y que sus actividades } \\
\text { finalizan luego de ese momento. }\end{array}$ \\
\hline
\end{tabular}

Nota: Elaboración propia

testimonios, se revelan detalles importantes sobre los efectos de los contenidos en dichos grupos poblacionales, sobre todo cuando un exempleado decide denunciar la situación, pues según sus declaraciones "...Facebook prioriza repetidamente los beneficios sobre la seguridad de los grupos poblacionales" (Hao. K, 2021)
Está claro que la historia de las redes sociales no está terminada y no es neutral (Frenkel. S., Kang. C. 2021). En esta línea la Comisión de Regulación de Comunicaciones (CRC, 2020), ha publicado su informe sobre la relación de los medios audiovisuales en dichas poblaciones, el cual tiene una caracterización importante en relación con la
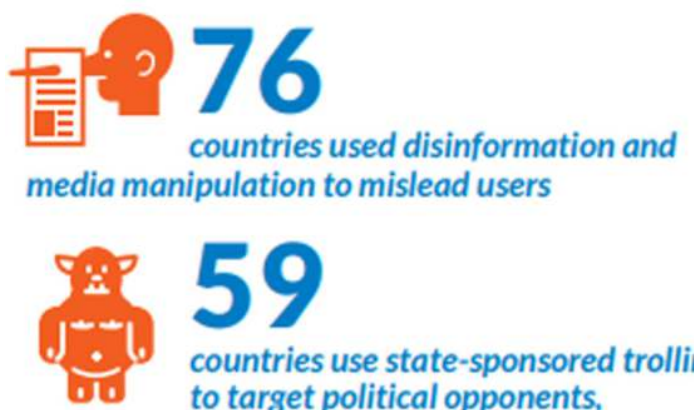

countries use state-sponsored trolling to target political opponents, activists or journalists

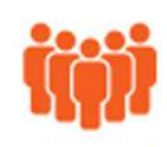
content \& accounts

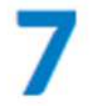

countries use mass-reporting of

Figura 9. Uso de estrategias. Fuente: https://demtech.oii.ox.ac.uk/wpcontent/uploads/sites/127/2021/02/CyberTroop-Report20-Draft9.pdf 


\begin{tabular}{|c|c|c|c|c|c|c|}
\hline & 今 & ఏ & 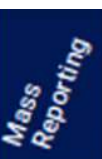 & 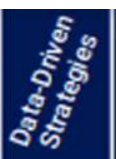 & s & 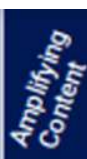 \\
\hline 2 & Angola & 72 & intilit & iiiiil & & 89 \\
\hline E= & Argentina & $\mathrm{R}^{2}$ & itipii & iiiiil & 밤 & ? \\
\hline 业 & Armenia & 7 & inifi & iniiil & y & 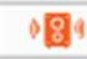 \\
\hline${ }^{2}=$ & Australia & 7 ? & ivitit & iiiiii & 항 & 9 \\
\hline & Austria & 70 & itili & iiiiil & y & 48 \\
\hline & Azerbaijan & $\mathrm{R}^{2}$ & itepii & iniiil & $y$ & 29 \\
\hline & Bahrain & 70 & Iilifi & iiiiii & 함 & 089 \\
\hline & Belarus & 70? & ififili & iiiiii) & 줍 & 2 (8) \\
\hline & Bolivia & $R^{2}$ & bith & 巢iii & 9 & : \\
\hline$\$ 1$ & Bosnia \& Herzegovina & $\mathrm{R}^{2}$ & itifil & iiiiii & 9 & 回 \\
\hline$\theta$ & Brazil & $R^{2}$ & itifiti & iiiiii & 출 & ? \\
\hline$=$ & Cambodia & $\mathrm{R}^{2}$ & intifi & iiiiii & 19 & : \\
\hline & China & $\mathrm{R}^{9}$ & itipif & 望ii & $y$ & 8 \\
\hline 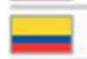 & Colombia & $77^{2}$ & tiliti & initi & 암 & 8 \\
\hline & Costa Rica & 8 & intifi & iniiil & yㅜㄹ & 48 \\
\hline 종 & Croatia & $\mathrm{R}^{2}$ & ilifi & iiiiil & $y$ & 08 \\
\hline$\approx$ & Cuba & $\mathrm{E}^{2}$ & biifi & iiniii & $y$ & ? \\
\hline$\square$ & Czech Republic & $E^{2}$ & initif & iiiiil & 함 & 89 \\
\hline 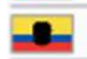 & Ecuador & $\mathrm{R}^{2}$ & tiliti & 唯 & 9 & (8) \\
\hline 플 & Egypt & 7 굴 & filifi & iiviii & 9 & (2) \\
\hline 틀 & El Salvador & $i^{2}$ ? & diliti & iiiii) & $y$ & 8 \\
\hline
\end{tabular}

Figura 10. Países que usan las técnicas. Fuente: https://demtech.oii.ox.ac.uk/wpcontent/uploads/sites/127/2021/02/CyberTroop-Report20-Draft9.pdf

definición de la población de (NN- los 6 a los 17 años, distribuidos en A), su demografía. El estudio re- tres categorías, (Figura 12). salta la forma de consumo de información a través de los distintos medios visuales. Para el efecto de esta investigación se revisan los que están asociados a los ecosistemas digitales.

El estudio define la población de estudio en relación con la edad de

Lo primero que resalta el estudio es que, en los hogares colombianos de dichas poblaciones, el acceso a los contenidos se puede realizar de distintas maneras. Indica también que hay un nivel alto de exposición a los contenidos, sin diferencia de estratos socioculturales de la po- 


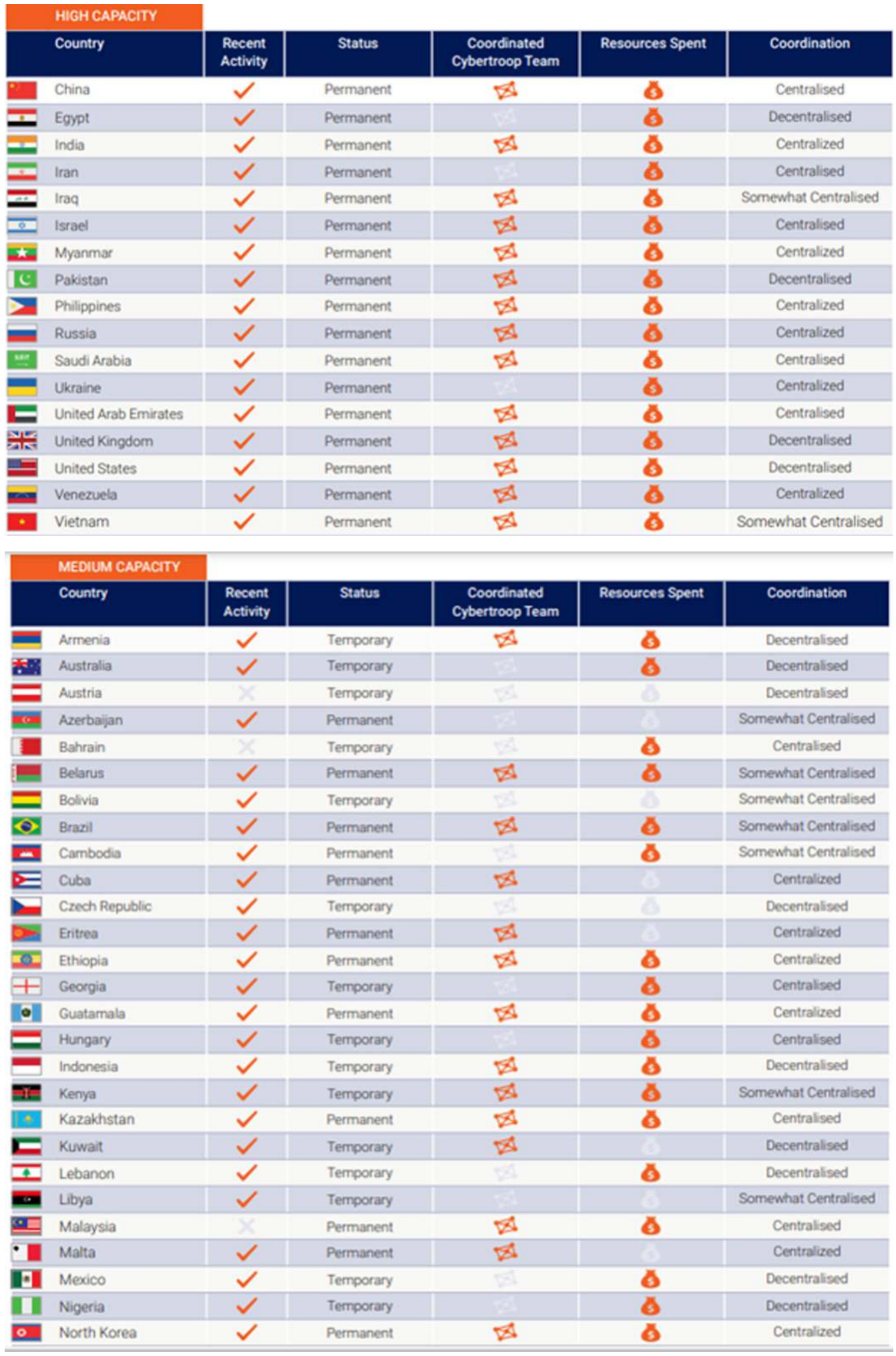




\begin{tabular}{|c|c|c|c|c|c|c|}
\hline & \multicolumn{6}{|l|}{ LOW CAPACTYY } \\
\hline & Country & $\begin{array}{l}\text { Recent } \\
\text { Activity }\end{array}$ & Status & $\begin{array}{c}\text { Coordinated } \\
\text { Cybertroop Team }\end{array}$ & Resources Spent & Coordination \\
\hline E & Angola & $x$ & Temporary & $\nabla$ & 8 & Decentralised \\
\hline-0 & Argentina & $\checkmark$ & Temporary & $\otimes$ & 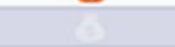 & Decentralised \\
\hline $\mathbf{N I}$ & Bosnia \& Herzegovina & $\checkmark$ & Temporary & E & 6 & Decentralised \\
\hline- & Colombia & $\checkmark$ & Temporary & $\otimes$ & 6 & Decentralised \\
\hline ” & Costa Rica & $\checkmark$ & Temporary & $\Delta$ & 8 & Decentralised \\
\hline$=$ & Croatia & $\checkmark$ & Temporary & $8=$ & 6 & Decentralised \\
\hline 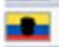 & Ecuador & $\checkmark$ & Temporary & 7 & 8 & Centralised \\
\hline 포 & El Salvador & $\checkmark$ & Temporary & Fe & 3 & Decentralised \\
\hline$=$ & Germany & $\checkmark$ & Temporary & $x^{4}$ & 8 & Decentralized \\
\hline \pm & Ghana & $\checkmark$ & Temporary & $\otimes$ & 6 & Centralized \\
\hline 㭋 & Greece & $\checkmark$ & Temporary & $\Delta$ & 8 & Decentralised \\
\hline III & Honduras & $\checkmark$ & Temporary & 2 & 28 & Centralised \\
\hline II & Italy & $\checkmark$ & Temporary & 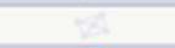 & 8 & Sornewhat Centralised \\
\hline 8 & Kyrgyzstan & $x$ & Temporary & $\Delta$ & 8 & Somewhat Centralised \\
\hline SE & Macedonia & $\checkmark$ & Temporary & 8 & 8 & Decentralised \\
\hline$[\bullet$ & Moldova & $\checkmark$ & Temporary & a & 8 & Decentralized \\
\hline$=$ & Netherlands & $\checkmark$ & Temporary & 2 & 0 & Decentralised \\
\hline$\square$ & Oman & $x$ & Temporary & a & as & Somewhat Centralised \\
\hline 口 & Qatar & $\checkmark$ & Temporary & 73 & 8 & Centralised \\
\hline 탕 & Serbia & $\checkmark$ & Permanent & $\Delta$ & 8 & Centralized \\
\hline$\equiv$ & South Africa & $\checkmark$ & Temporary & $\Delta$ & 8 & Centralized \\
\hline 드 & Spain & $\checkmark$ & Temporary & 20 & 3 & Decentralised \\
\hline$=$ & Sudan & $\checkmark$ & Temporary & $\otimes$ & 6 & Centralized \\
\hline 를 & Sweden & $\checkmark$ & Temporary & 20 & 6 & Decentralised \\
\hline$\theta$ & Tunisia & $\checkmark$ & Temporary & 7 & 8 & Somewhat centralised \\
\hline$=$ & Uzbekistan & $\checkmark$ & Permanent & $\Delta$ & 5 & Centralised \\
\hline$=$ & Zimbabwe & $\checkmark$ & Temporary & 28 & 0 & Centralised \\
\hline
\end{tabular}

Figura 11. Capacidades distribuidas por países. Fuente: https://demtech.oii.ox.ac.uk/wpcontent/uploads/sites/127/2021/02/CyberTroop-Report20-Draft9.pdf

blación en estudio. Se establece que hay una disponibilidad de 3.4 tipos de dispositivos distintos; el $83 \%$ de los (NNA) mencionan disponibilidad de un teléfono inteligente en el hogar (Figura 13).

El consumo de los contenidos y el uso de Internet en estos grupos poblacionales está distribuido de acuerdo con las edades, siendo los adolescentes los que más uso de Internet tienen (Figura 14).
El uso de las redes sociales es la tercera actividad que más realizan los (NNA), con un $55 \%$ y dentro de dicho consumo las redes sociales usadas son, Facebook, Instagram, Twitter y TikTok (18\%) Así mismo, se identifica que el acceso a los contenidos se registra en gran medida en los momentos de las comidas; chatear por celular el 38\%; interactuar en redes sociales $30 \%$, comportamiento más marcado en los grupos de adolescentes. 


\section{LA POBLACIÓN BAJO ESTUDIO SE CARACTERIZA POR}

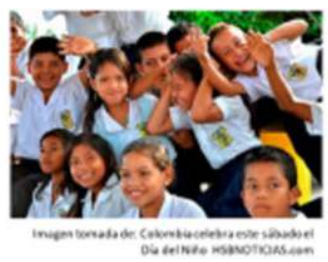

NIÑEZ 6-9 AÑOS

$\checkmark$ desarRollo autonomo

$\checkmark$ constantes cambios

$\checkmark$ adultos que garanticen su desarrollo

$\checkmark$ desarrollo de nuevas destrezas DEPENDE dE FACTORES GENÉticos, NUTRICIONALES, SOCIALES

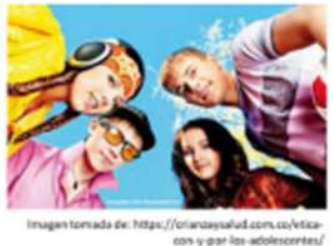

PREADOLESCENCIA 10-13 AÑOS

$\checkmark$ existencia del egocentrismo

$\checkmark$ CAMBIOS CORPorales Y APARIENCIA Fisica

cumpur expectativas Sociales,

$\checkmark$ PONe A PRUEBa la Autoridad y los PARES COBRAN MAYOR IMPORTANCIA.

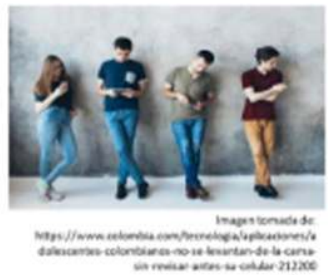

ADOLESCENCIA 14-17AÑOS

distanciamiento afectivo de la FAMILIA

$\checkmark$ acercamiento al grupo de pares,

$\checkmark$ PROPIOS JUICIOS Y VALLRES

$\checkmark$ PENSAMIENTO CRITICO

$\checkmark$ INVOLUCRAMIENTO PARES INTENSO

Figura 12. Población de Estudio. Fuente:

https://www.crcom.gov.co/uploads/images/files/2021/INFORME\%20FINAL\%20CON SULTOR\%20ESTUDIO\%20DE\%20INFANCIA\%20DIC_2020.pdf

En cuanto a consumo de plataformas digitales, se tiene que los menores entre 6 y 9 años consumen menos contenidos que las otras dos franjas poblacionales (Figura 15). Siendo Youtube, Netflix y Fa- cebook las tres plataformas digitales principalmente usadas para interactuar con contenidos digitales.

El consumo del contenido en las distintas redes sociales ocurre en 6

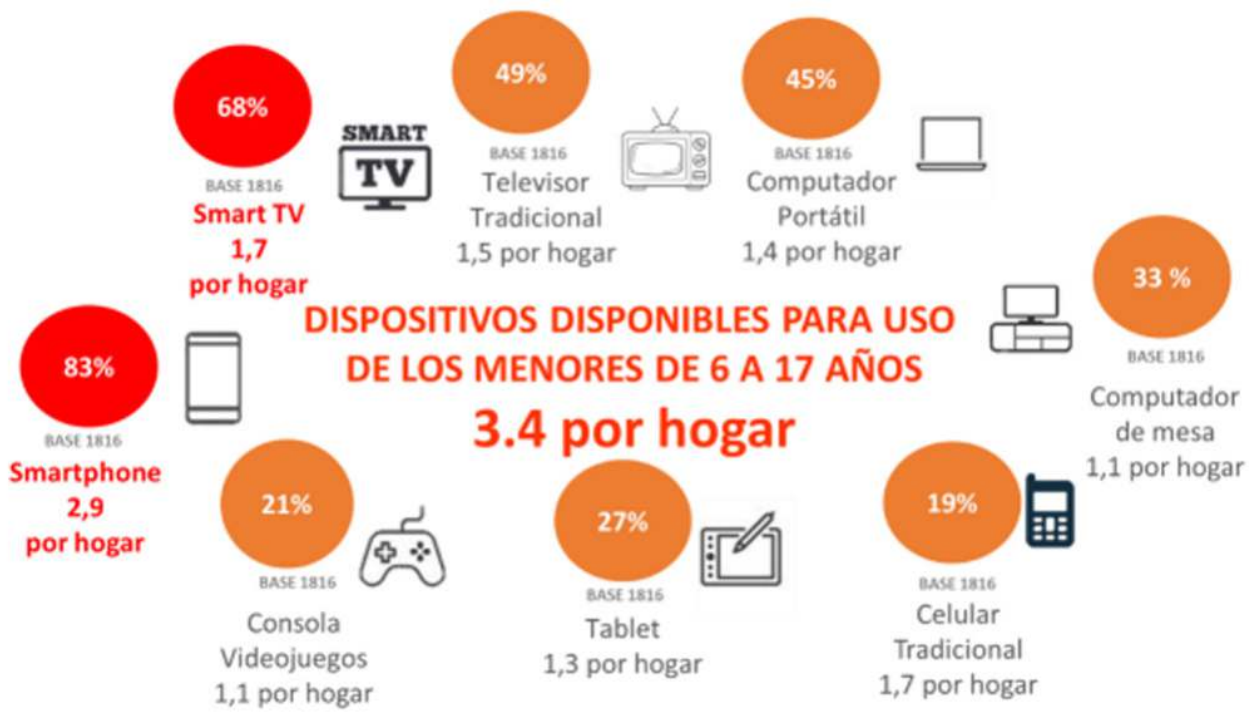

Figura 13. Distribución de los medios disponibles. Fuente:

https://www.crcom.gov.co/uploads/images/files/2021/INFORME\%20FINAL\%20CON SULTOR\%20ESTUDIO\%20DE\%20INFANCIA\%20DIC_2020.pdf 


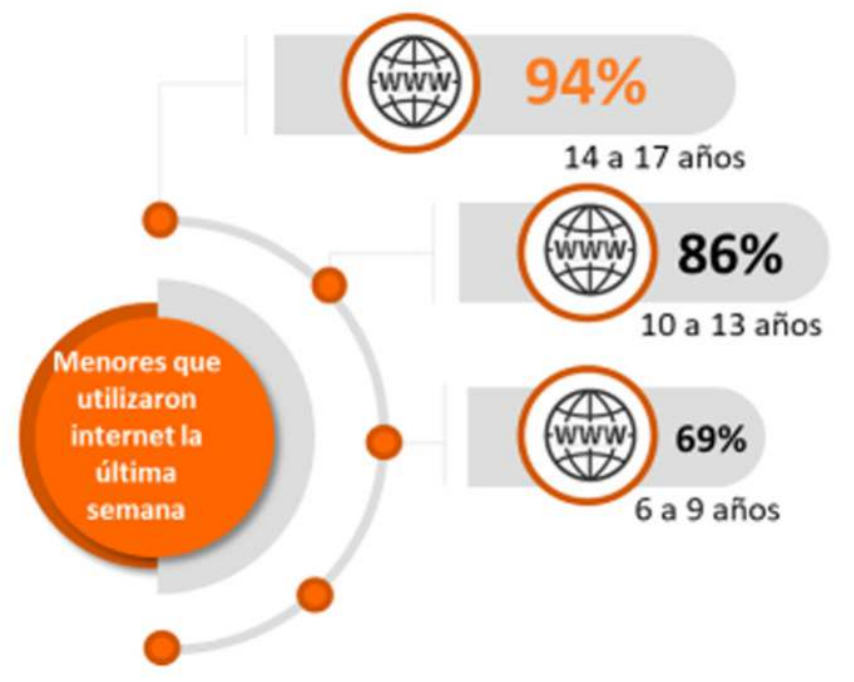

Figura 14. Distribución del uso de Internet. Fuente: https://www.crcom.gov.co/uploads/images/files/2021/INFORME\%20FINAL\%20CON SULTOR\%20ESTUDIO\%20DE\%20INFANCIA\%20DIC_2020.pdf

de cada 10 niños, el aumento de la los datos relevantes del estudio se edad es un factor proporcional al destaca que este grupo poblacional aumento del consumo, comparado en general ve en el acceso a Intercon el uso de Internet para hacer ta- net una forma de interacción; el reas, el cual es bastante bajo. De $93 \%$ manifiesta disfrutar conectar-

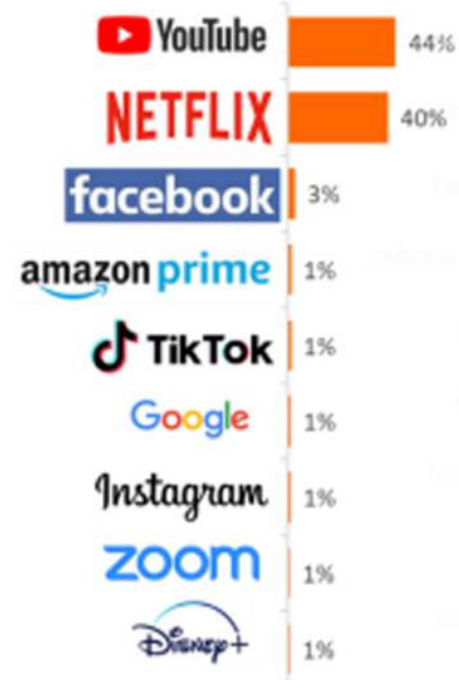

Figura 15. Recordación de plataformas digitales. Fuente:

https://www.crcom.gov.co/uploads/images/files/2021/INFORME\%20FINAL\%20CON

SULTOR\%20ESTUDIO\%20DE\%2OINFANCIA\%20DIC_2020.pdf 
se a internet; el $79 \%$ manifiesta que es un medio con el que tiene cercanía y solo el $34 \%$ manifiesta que confía en el contenido que ahí se encuentra. Sin embargo, el 53\% manifiesta que está muy pendiente de lo que allí se publica.

El comportamiento de los (NNA) es muy variado, el estudio indica que hay múltiples comportamientos asociados al consumo de contenidos y resalta que, solo el $58 \%$ habla de los contenidos que consume, el $41 \%$ comparte contenidos, el $71 \%$ sigue a influencers, personajes $y / 0$ canales, el $57 \%$ piensa en lo que ve en Internet, solo el $41 \%$ encuentra relación entre lo que vive y los contenidos que ve en Internet, el 64\% manifiesta que algunas veces no entiende lo que ve en Internet y solo el $27 \%$ manifiesta subir contenido.

Otro de los datos interesantes del estudio es el término "prosumo", que se refiere a las personas con interés en producir y generar contenidos para los distintos medios de comunicación. En tal sentido, indica que los (NNA) no tienen la tendencia a ser prosumidores, sino más bien consumidores; no obstante, se empieza a ver la tendencia de algunos por empezar a explorar el prosumo como una forma de interacción con otros.

Lo importante que se debe contemplar no es solo el uso de las redes sociales, sino sus efectos; existe un lado oscuro de todo el uso de medios sociales, que puede ser a- grupado en seis categorías importantes, como lo señala (Boroon. L., Abedin. B., 2021)

Costos del intercambio social: incluye tanto los daños psicológicos, como la depresión, la ansiedad o los celos, y otros costos como la pérdida de tiempo, energía y dinero.

Contenido molesto: incluye una amplia gama de contenido que molesta, molesta o irrita, como contenido perturbador o violento o contenido sexual u obsceno.

Problemas de privacidad: incluye cualquier amenaza a la privacidad personal relacionada con el almacenamiento, la reutilización o el intercambio de información personal con terceros.

Amenazas de seguridad: se refiere a daños por fraude o engaño, como phishing o ingeniería social.

Ciberacoso: incluye cualquier abuso o acoso por parte de grupos o individuos, como mensajes abusivos, mentiras, acecho o difusión de rumores.

Bajo rendimiento: se refiere al impacto negativo en el desempeño laboral o académico.

Resaltando que algunas de estas categorías tienen amplia incidencia en las poblaciones de (NNA), y que una educación y mayor consciencia de los peligros potenciales pue- 
de aumentar la moderación de las personas en su uso e impactar a todo el ecosistema, para disminuir los efectos negativos, según los investigadores.

\section{La desinformación}

Existen muchas clases de tratamientos de la información, que gracias al poder de los algoritmos se pueden presentar. Muestra tres categorías documentadas sobre el uso inadecuado de la información (UNESCO, 2020).

Desinformación (disinformation): Información que es falsa y se distribuye de manera intencional para crear daño a una persona, grupo, organización o país.

Información maliciosa (mal-information): Información basada en la realidad que es usada para crear daño a una persona, grupo, organización o estado.

Información errónea (misinformation): Información errónea, que no tiene como propósito o no fue creada para hacer daño o mala intención.

Las tres fuentes relacionadas con el manejo de la información son cruciales, pues en la actualidad son factores esenciales para entender dentro de la potencia de los algoritmos. La figura 16 muestra lo anteriormente mencionado. A pesar de su distinción, las consecuencias sobre las sociedades pueden tener efectos similares y devastadores.

La manipulación social hostil es definida como "...la generación y difusión intencionada y sistemática de información para producir resulta-

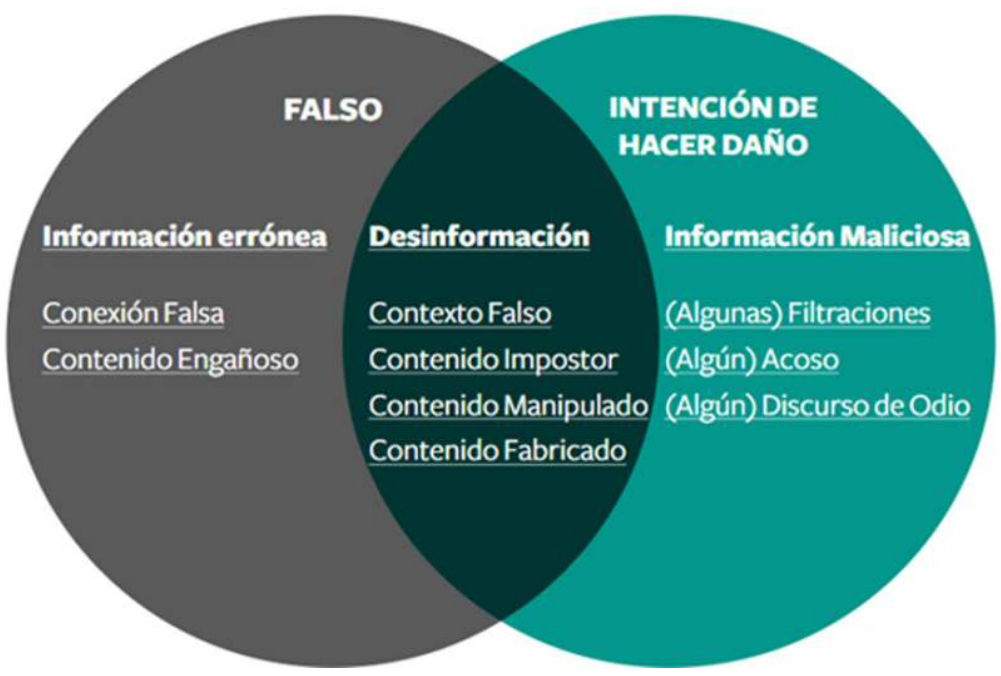

Figura 16. Desorden de la información. Fuente:

https://unesdoc.unesco.org/in/documentViewer.xhtml?v=2.1.196\&id=p::usmarcdef_00 00373349\&file=/in/rest/annotationSVC/DownloadWatermarkedAttachment/attach_imp ort_73d03993-db18-4ae9-8f64-de9a0d729c22\%3F_\%3D373349spa.pdf\& 
dos sociales, políticos y económicos perjudiciales en un país objetivo, afectando a las creencias, actitudes y comportamientos" (Mazarr. M., et all, 2019).

Muchos especialistas lo consideran un fenómeno de atención en el radar y en los ojos de las agendas globales, como un problema y desafío que puede generar guerras.

La revolución digital en la que actualmente se desenvuelve el mundo, lo global de las comunicaciones, la velocidad y fluidez de la información, la dependencia en las tecnologías ha creado el ambiente perfecto para que la manipulación a través de la desinformación esté presente (Elonheimo. T., 2021).
En un estudio realizado por The Pearson Institute y The Associated Press-NORC Center for Public Affairs (Pearson Institute/APNORC.2021) de la desinformación en Estados Unidos, encuentra que más del $90 \%$ de las personas creen que es un gran problema y muchos culpan a las redes sociales, a las personas y los partidos políticos de propender por que esto suceda.

Los datos muestran que la población en general sí está preocupada por el tema y que pasa en sus círculos sociales; sin embargo, se detalla que no se ven como parte del problema (Figura 17).

Para la población de estudio son las redes sociales en sí mismas y

\section{Many Americans are concerned about exposure to misinformation. \\ Percent of adults}

Extremely/very concerned $\quad$ a Somewhat concerned $\quad$ Not very/not at all concerned

have spread misinformation

I have been exposed to misinformation

My friends have spread misinformation

My friends have been exposed to misinformation

My family have spread misinformation

My family have been exposed to misinformation
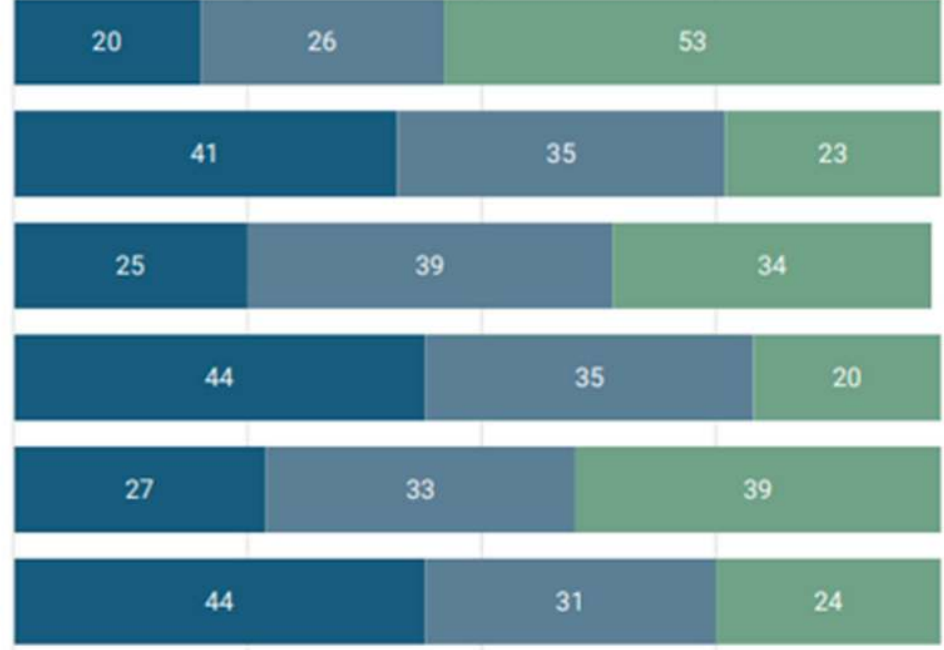

0

25

50

75

$100 \%$

Figura 17. Preocupación por la desinformación. Fuente: https://apnorc.org/wpcontent/uploads/2021/10/misinformation_Formatted_v2-002.pdf 
Most Americans say both social media companies and users are responsible for spreading misinformation.

Percent of adults

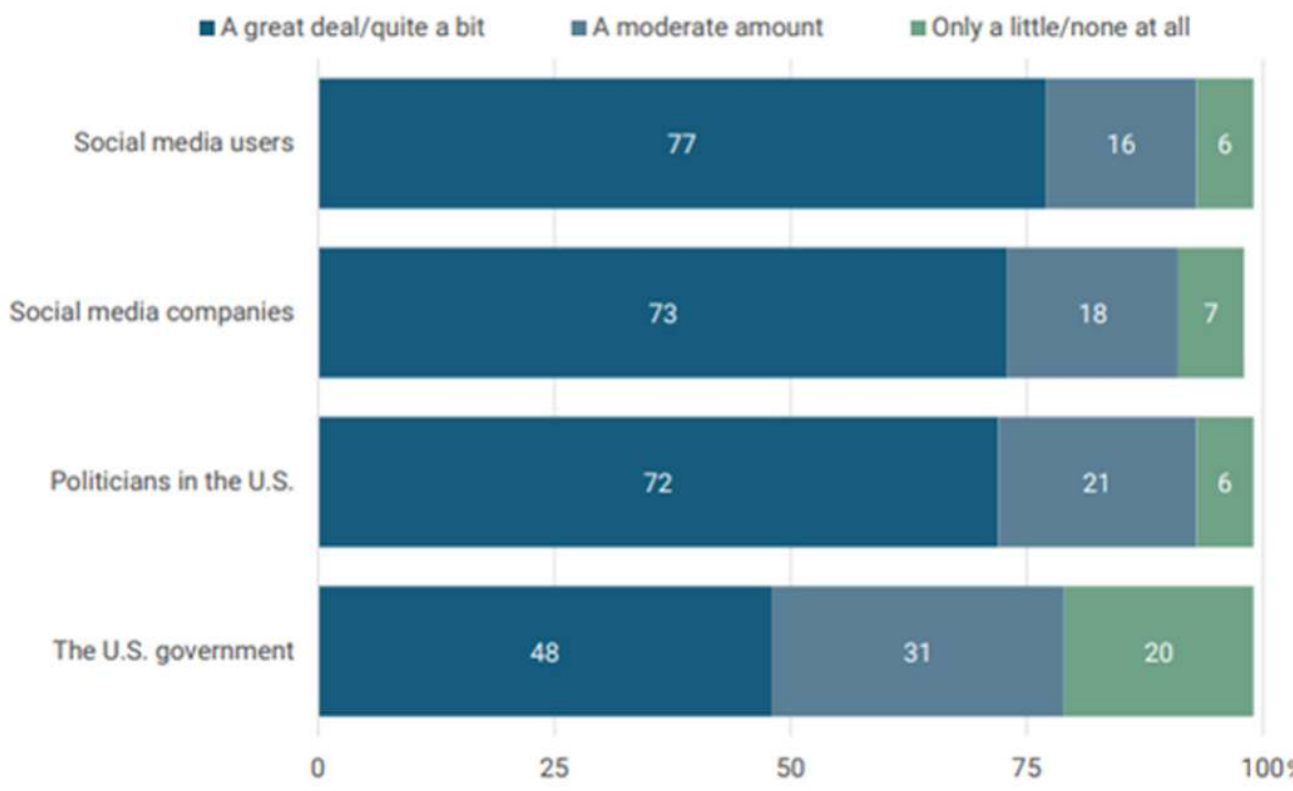

Figura 18. responsabilidad de la desinformación. https://apnorc.org/wpcontent/uploads/2021/10/misinformation_Formatted_v2-002.pdf

las personas que las usan, las responsables por este fenómeno (Figura 18). En tal sentido se considera a las personas más responsables, que las compañías que prestan estos servicios.

Así mismo, al indagar por quiénes pueden ser responsables, la población en estudio considera que países como China, Rusia y otras naciones, están detrás de estos fenómenos que afectan a la sociedad en general (Figura 19). La desinformación es un fenómeno de vieja data, no obstante, las deepfakes y la facilidad de difundir el contenido en línea, lo hacen más propenso a que sean usados por los gobiernos y cualquier tipo de entidades (MIT, 2020).

El estudio advierte que se trata de un problema que debe ser enfrentado desde el ambiente gubernamental por lo que afecta de una manera directa la vida de las personas, aun así, consideran que la educación individual es clave para ejercer control a este fenómeno.

La desinformación también adquiere fuerza y poder como una amenaza digital, que pasa de ser latente y distante, a presente y recurrente; que usa como herramientas y armas a las deepfakes, la manipulación de videos, de audios y 


\section{About half of Americans say the Chinese and Russian governments are largely responsible for misinformation.}

Percent of adults

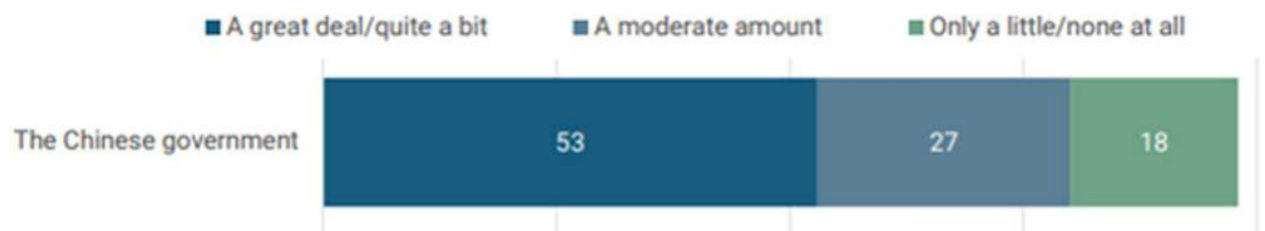

The Russian government

54

The Iranian government

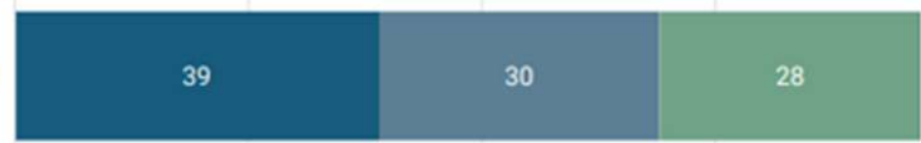

Other foreign governments

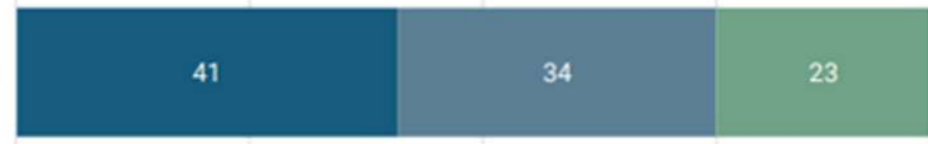

0

25

50

75

$100 \%$

Figura 19. Responsabilidad de gobiernos. https://apnorc.org/wpcontent/uploads/2021/10/misinformation_Formatted_v2-002.pdf

demás estrategias, que pueden crear inestabilidad en los estados, organizaciones y la población en general (Hill. M., 2021)

\section{Conclusiones}

Los algoritmos definitivamente han ganado espacio en la sociedad y en su expresión máxima son las aplicaciones su forma material de expresarse y tienen una influencia significativa en la vida de todos.

Al revisar estos informes en donde se evidencia la presencia de su es necesario repensar las formas en cómo se deben exponer las personas a su influencia, no se trata de que el algoritmo es el responsable del daño, sino del uso que se le da.
La desinformación es una industria formalizada, industrializada y muy profesional, producida a gran escala y que tiene claros actores, estrategias y propósitos claramente definidos (Bradshaw, S., et al., 2021).

Definitivamente los contenidos en estos espacios tienen gran presencia y efecto en la vida de las personas, especialmente en los ( NNA); la facilidad de uso, la amplitud del contenido y la posibilidad de acceso son elementos que en Colombia tienen relevancia a la hora del consumo del contenido. El Covid19 es un factor fundamental que aceleró el consumo de contenidos en dichas poblaciones, convirtien- 
do los contenidos digitales en permanentes y cotidianos; por tanto, se requiere de una educación en dichos aspectos (CRC,2020).

El año 2016 se ha convertido en un punto de giro para entender la desinformación y el poder de los algoritmos como algo más complejo que ha tomado por sorpresa a gobiernos, entidades y organizaciones. La evolución de las técnicas, tácticas y estrategias de la desinformación, sumado a la evolución de las tecnologías como la inteligencia artificial traerán grandes desafíos en la sociedad (CBINSIHGTS, 2020).

Es importante considerar el diseño de los algoritmos y sus efectos en las personas, para prevenir propósitos nefastos (Nitzberg. M. 2021).

Definitivamente el presente y futuro de la confianza en las organizaciones está en una gran tensión, toda vez que la desinformación, los cambios en la información y los distintos intereses continúan siendo una constante. También es cierto que las personas necesitan encontrar mecanismos que les ayuden a estar alerta en medio del ruido digital actual (Sandal. G., 2021).

Definitivamente con el auge y aumento del uso del algoritmo como herramienta para crear nuevos y mejorados servicios que penetren más en la vida digital de los seres humanos, es necesario una mayor alfabetización digital, como meca- nismo para contrarrestar el mal uso de dichos algoritmos.

Sin una conciencia ética de cómo se quieren usar los algoritmos, y cuál debe ser su para qué o razón, podemos sucumbir y quedar ciegos, ante su potencial y los usos que estos nos ofrecen (Cremer. D., 2020), sin dejar de lado la posibilidad de caer en las garras de un uso inadecuado de los mismos.

Hacer cada vez más escrutinio de los algoritmos, y que estos tengan más observabilidad será uno de los grandes desafíos en ambientes como los actuales, sometidos y gobernados cada vez más su poder y su uso. En la misma medida es necesario que todas las partes entiendan los impactos sociales que estos poseen y que debe ser materia de continuo estudio y verificación (Beer. D., 2017).

Definitivamente sin ser la única solución ni la más exacta posible, la educación en los datos (Carmi. E., 2019), el uso o lo que se llama la alfabetización digital a gran escala, podría ser una herramienta que ayude a contrarrestar los efectos complejos que están teniendo los usos inadecuados de los algoritmos y su exposición en la vida cotidiana.

En la era de la desinformación como nueva forma de conflicto (Mazarr. M., 2019), es necesario hacer grandes esfuerzos por acompañar a todos los actores, y crear redes 
de cooperación entre todas las partes, gobiernos, sociedad civil, empresas públicas y privadas, que permitan aprender el uso adecuado de la información y diseñar agendas de cooperación para enfrentar una compleja situación en nuestros tiempos.

\section{Referencias}

Abiteboul, S., \& Dowek, G. (2020). The Age of Algorithms. Cambridge:

Cambridge University Press.

DOI:10.1017/9781108614139

Beer. D. (2017) The social power of algorithms. Information, Communication \& Society. 20(1). 1-13, DOI:

10.1080/1369118X.2016.1216147

Boroon. L. \& Abedin. B. (2021). The Dark Side of Using Online Social Networks:

A Review of Individuals' Negative Experiences. DOI:

10.4018/JGIM.20211101.oa34.

https://www.igi-global.com/article/thedark-side-of-using-online-social-net works/276942

Bradshaw, S., Bailey, H., \& Howard, P. (2021). Industrialized Disinformation: 2020 Global Inventory of Organized Social Media Manipulation. Oxford, UK: Programme on Democracy \& Technology.

https://demtech.oii.ox.ac.uk/research/ posts/industrialized-disinformation/

Carmi. E., (2019). Educarnos en información, para evitar estar desinformados. OpenDemocracy.

https://www.opendemocracy.net/es/de mocraciaabierta-es/educarnos-eninformaci\%C3\%B3n-para-evitar-estardesinformados/

CBINSIGHTS. (2020). Disinformation That Kills: The Expanding Battlefield Of Digital Warfare.
https://www.cbinsights.com/reports/C B-Insights_Future-of-InformationWarfare.pdf

CRC. (2020). "Infancia, adolescencia y medios audiovisuales en Colombia: apropiación, usos y actitudes".

Comisión de Regulación de Comunicaciones.

https://www.crcom.gov.co/uploads/ima ges/files/2021/informe $\% 20$ final\%20co nsultor\%20estudio\%20de\%20infancia \%20dic_2020.pdf

Cremer. D. (2020). Leadership by Algorithm. Who Leads and who follows in the Al era?. Hampshire, UK. Harriman House Ltd.

Elonheimon. T. (2021). Comprehensive Security Approach in Response to Russian Hybrid Warfare. Strategic studies quarterly 13(5).

https://www.airuniversity.af.edu/Portal s/10/S SQ/documents/Volume15_Issue-3/Elonheimo.pdf

Frenkel. S., Kang. C. (2021). The Ugly Truth. Inside Facebook's battle for domination. New York. USA.

HarperCollins Publisher.

Hill. M. (2021). How deepfakes enhance social engineering and authentication threats, and what to do about it.

https://www.csoonline.com/article/363 6992/how-deepfakes-enhance-socialengineering-and-authentication-thre ats-and-what-to-do-about-it.html

Hao. K. (2021). Claves del testimonio de la denunciante de Facebook en el Senado de EE. UU. MIT Technology Review.

https://www.technologyreview.es/s/13 714/claves-del-testimonio-de-la-de nunciante-de-facebook-en-el-senadode-ee-uu

Nitzberg. N. (2021). Why 'Explicit Uncertainty' Matters for the Future of Ethical Technology. MIT Sloan Management. 
https://sloanreview.mit.edu/article/why -explicit-uncertainty-matters-for-thefuture-of-ethical-technology/

MIT. (2020). MIT Center for Advanced Virtuality. Tackling the misinformation epidemic with "In Event of Moon Disaster".

https://news.mit.edu/2020/mit-tacklesmisinformation-in-event-of-moondisaster-0720

Mazarr, M., Bauer. R, Casey. A, Heintz. S. \& Matthews. L. (2019). The Emerging Risk of Virtual Societal Warfare: Social Manipulation in a Changing Information Environment. Santa Monica, CA:

RAND Corporation.

https://www.rand.org/pubs/research_r eports/RR2714.html

Pearson Institute/AP-NORC. (2021). THE AMERICAN PUBLIC VIEWS THE SPREAD OF MISINFORMATION AS A MAJOR PROBLEM. The Pearson Institute, The Associated Press-NORC Center for Public Affairs.

https://apnorc.org/wp-content/up loads/2021/10/misinformation_Format ted_v2-002.pdf
Sandal. G. (2021). Future of Truth in the Information Age? Future Plataform. https://www.futuresplatform.com/blog/f uture-truth-information-age

Sadowski, Jathan [2020], Too Smart. How Digital Capitalism Is Extracting Data, Controlling Our Lives, and Taking Over the World, Cambridge, MIT Press.

Soler, E. (2020). El mundo en 2021: diez temas que marcarán la agenda internacional. CIDOB Notes Internationals. 243.

https://www.cidob.org/publicaciones/s erie_de_publicacion/notes_internacio nals_cidob/243/el_mundo_en_2021_d iez temas que marcaran la ${ }^{-}$agenda internacional

UNESCO. (2020). PERIODISMO, "NOTICIAS FALSAS" \& DESINFORMACIÓN.

https://unesdoc.unesco.org/ark:/4822 3/pf0000373349

WSJ. (2021). The Facebook Files. Washintong Post.

https://www.wsj.com/articles/thefacebook-files-11631713039

Andrés R. Almanza J., Ms.C, CISM. Chief Growth Officer en CISOS.CLUB, Investigador en Ciberseguridad SegInfo y Liderazgo. Executive Certificate in Cybersecurity Leadership \& Strategy by FIU University, Certificado como ISO 27001 Lead Implementer and 27005 Lead Manager from PECB, CISM, ITILv3, LPI, Certificado como Coach Profesional Internacional, Máster in Leadership and Organizational Development with Coaching, Executive Master's in Leadership Skills Developed in Harvard, \& Coach, Profesional avalado por International Coach Federation; Profesional en Ingeniería de Sistemas, especialista en seguridad en redes y máster en Seguridad de la Información. Docente del programa de maestría de la Universidad Externado de Colombia y de la Universidad de las Américas en Ecuador. Creador de la Comunnidad CISOS.CLUB, CISOS-COL y CISOS-LATAM (Linkedin) y Miembro del Comité Editorial de la revista "Sistemas" de ACIS. 